\title{
Mathematical expressions for quantum fluctuations of energy for different energy-momentum tensors
}

\section{Rajeev Singh*}

Institute of Nuclear Physics Polish Academy of Sciences, PL-31-342 Kraków, Poland

E-mail: rajeev.singh@ifj.edu.pl

Expressions for the quantum fluctuations of energy density have been derived for the subsystems consisting of hot relativistic gas of particles with spin- $\frac{1}{2}$ and mass $m$. Our expressions for the fluctuation depend on the form of energy-momentum tensor which in turn depends on the choice of pseudo-gauge. These results suggest that quantum fluctuations of energy should be considered seriously in the case of very small thermodynamic systems.

The Ninth Annual Conference on Large Hadron Collider Physics-LHCP2021

7-12 June, 2021

online

${ }^{*}$ Speaker 


\section{Introduction}

Quantum and statistical fluctuations intrinsic to any many-body system [1] have an important role as they contain crucial information about the possible phase transitions [1-15], dissipative phenomena [16-34], and the formation of the large scale structures in the universe [35-43]. Following the footsteps of our previous works [44-49], we analyze and study the pseudo-gauge dependence on the quantum fluctuations of energy, which means, using the pseudo-gauge transformation we can choose different form of the energy-momentum tensor for the description of the system. Any energy-momentum tensor $\hat{T}^{\mu \nu}$ satisfying the conservation equation $\partial_{\mu} \hat{T}^{\mu \nu}=0$ can be used to construct a new conserved energy-momentum tensor as [50-52]

$$
\hat{T}^{\prime \mu \nu}=\hat{T}^{\mu \nu}+\partial_{\lambda} \hat{A}^{v \mu \lambda} \quad \text { with } \quad \hat{A}^{v \mu \lambda}=-\hat{A}^{v \lambda \mu} .
$$

For the current analysis, we consider a system of spin- $\frac{1}{2}$ particles and study the effects of different pseudo-gauges. We choose three different forms of energy-momentum tensor, such as canonical form from the Noether theorem [53-55], the de Groot-van Leeuwen-van Weert (GLW) form [56], and the Hilgevoord-Wouthuysen (HW) form [57, 58]. These forms are currently being discussed widely in the context of heavy-ion collisions for the study of spin polarization [52, 59], as the pseudo-gauge choices can also be applied for the spin tensor $\hat{S}^{\lambda, \mu \nu}$ which is a part of the total angular momentum tensor $\hat{J}^{\lambda, \mu \nu}=\hat{L}^{\lambda, \mu \nu}+\hat{S}^{\lambda, \mu \nu}[51,52,60-64]$. We calculate the quantum fluctuations of the $\hat{T}^{00}$ component of the energy-momentum tensor and find that even though $\hat{T}^{00}$ depends on pseudo-gauge, its thermal average value is independent of it. In addition, we see that for small size subsystems the fluctuations are pseudo-gauge dependent but become independent if the size of the system is large. This analysis might be useful for the understanding of the concept of energy-density in the context of relativistic heavy-ion collisions [65-76]. Our conclusion is that the quantum fluctuations of energy-density have no physical significance in small size subsystems and a specific pseudo-gauge must be chosen in order to describe the system.

\section{Basic definitions}

As in our previous studies [44-46], here we assume a subsystem $S_{a}$ inside a larger thermodynamic system $S_{V}$ consisting of spin- $\frac{1}{2}$ particles having mass $m$ with no conserved charges. The volume $V$ of the system $S_{V}$ is large enough to perform integrals over particle momentum. ${ }^{1} \mathrm{We}$ describe our system by a spin- $\frac{1}{2}$ field in thermal equilibrium where the field operator is [77]

$$
\psi(t, \boldsymbol{x})=\sum_{r} \int \frac{d^{3} k}{(2 \pi)^{3} \sqrt{2 \omega_{\boldsymbol{k}}}}\left(U_{r}(\boldsymbol{k}) a_{r}(\boldsymbol{k}) e^{-i k \cdot x}+V_{r}(\boldsymbol{k}) b_{r}^{\dagger}(\boldsymbol{k}) e^{i k \cdot x}\right)
$$

with $a_{r}(\boldsymbol{k})$ and $b_{r}^{\dagger}(\boldsymbol{k})$ being the annihilation and creation operators for particles and antiparticles, respectively, satisfying the anti-commutation relations, $\left\{a_{r}(\boldsymbol{k}), a_{s}^{\dagger}\left(\boldsymbol{k}^{\prime}\right)\right\}=(2 \pi)^{3} \delta_{r s} \delta^{(3)}\left(\boldsymbol{k}-\boldsymbol{k}^{\prime}\right)$ and $\left\{b_{r}(\boldsymbol{k}), b_{s}^{\dagger}\left(\boldsymbol{k}^{\prime}\right)\right\}=(2 \pi)^{3} \delta_{r s} \delta^{(3)}\left(\boldsymbol{k}-\boldsymbol{k}^{\prime}\right)$. The index $r$ is the polarization degree of freedom, and $U_{r}(\boldsymbol{k})$ and $V_{r}(\boldsymbol{k})$ are the Dirac spinors where $\omega_{\boldsymbol{k}}=\sqrt{\boldsymbol{k}^{2}+m^{2}}$ is the energy of a particle.

\footnotetext{
${ }^{1}$ Metric $g_{\mu \nu}=\operatorname{diag}(+1,-1,-1,-1)$ is used. Three-vectors are shown in bold font and a dot is used to denote the scalar product of both four- and three-vectors, i.e., $a^{\mu} b_{\mu}=a \cdot b=a^{0} b^{0}-\boldsymbol{a} \cdot \boldsymbol{b}$.
} 
The following expectation values are required to calculate thermal averages [78-80] of the energy-density operator $\hat{T}_{a}^{00}$

$$
\begin{aligned}
& \left\langle a_{r}^{\dagger}(\boldsymbol{k}) a_{s}\left(\boldsymbol{k}^{\prime}\right)\right\rangle=(2 \pi)^{3} \delta_{r s} \delta^{(3)}\left(\boldsymbol{k}-\boldsymbol{k}^{\prime}\right) f\left(\omega_{\boldsymbol{k}}\right), \\
& \left\langle a_{r}^{\dagger}(\boldsymbol{k}) a_{s}^{\dagger}\left(\boldsymbol{k}^{\prime}\right) a_{r^{\prime}}(\boldsymbol{p}) a_{s^{\prime}}\left(\boldsymbol{p}^{\prime}\right)\right\rangle=(2 \pi)^{6}\left(\delta_{r s^{\prime}} \delta_{r^{\prime} s} \delta^{(3)}\left(\boldsymbol{k}-\boldsymbol{p}^{\prime}\right) \delta^{(3)}\left(\boldsymbol{k}^{\prime}-\boldsymbol{p}\right)\right. \\
& \left.-\delta_{r r^{\prime}} \delta_{s s^{\prime}} \delta^{(3)}(\boldsymbol{k}-\boldsymbol{p}) \delta^{(3)}\left(\boldsymbol{k}^{\prime}-\boldsymbol{p}^{\prime}\right)\right) f\left(\omega_{\boldsymbol{k}}\right) f\left(\omega_{\boldsymbol{k}^{\prime}}\right) .
\end{aligned}
$$

where $f\left(\omega_{\boldsymbol{k}}\right)$ is the Fermi-Dirac distribution function for particles. $\hat{T}_{a}^{00}$ is an energy-density operator expressed below of the subsystem $S_{a}$ which is placed at the origin of coordinate system [50], and we use Gaussian profile to define our subsystem $S_{a}$ in order to have no sharp-boundary effects

$$
\hat{T}_{a}^{00}=\frac{1}{(a \sqrt{\pi})^{3}} \int d^{3} \boldsymbol{x} \hat{T}^{00}(x) \exp \left(-\frac{\boldsymbol{x}^{2}}{a^{2}}\right) .
$$

Then, we calculate the variance $\left(\sigma^{2}\right)$ and the normalized standard deviation $\left(\sigma_{n}\right)$ using the expressions below in order to find the fluctuation of the energy density of the subsystem $S_{a}$,

$$
\sigma^{2}(a, m, T)=\left\langle: \hat{T}_{a}^{00}:: \hat{T}_{a}^{00}:\right\rangle-\left\langle: \hat{T}_{a}^{00}:\right\rangle^{2}, \quad \sigma_{n}(a, m, T)=\frac{\left(\left\langle: \hat{T}_{a}^{00}:: \hat{T}_{a}^{00}:\right\rangle-\left\langle: \hat{T}_{a}^{00}:\right\rangle^{2}\right)^{1 / 2}}{\left\langle: \hat{T}_{a}^{00}:\right\rangle}
$$

where $\left\langle: \hat{T}_{a}^{00}:\right\rangle$ is the thermal expectation value of $\hat{T}_{a}^{00}$ after doing normal ordering.

\section{Energy density fluctuation in different pseudo-gauges}

\subsection{Canonical framework}

The canonical form of energy-momentum tensor is given as [77]

$$
\hat{T}_{\text {Can }}^{\mu \nu}=\frac{i}{2} \bar{\psi} \gamma^{\mu \stackrel{\leftrightarrow}{\partial}}{ }^{v} \psi
$$

where the thermal expectation value of $\hat{T}_{\text {Can }, a}^{00}$ for the subsystem $S_{a}$ is calculated as

$$
\left\langle: \hat{T}_{\mathrm{Can}, a}^{00}:\right\rangle=4 \int \frac{d^{3} k}{(2 \pi)^{3}} \omega_{\boldsymbol{k}} f\left(\omega_{\boldsymbol{k}}\right) \equiv \varepsilon_{\mathrm{Can}}(T) .
$$

with the factor 4 representing the spin degeneracy $\left(g_{s}=(2 s+1)\right)$. The canonical energy-density $\varepsilon_{\text {Can }}(T)$, Eq. (8), is independent of both time and the system size $a$ indicating the system's spatial uniformity. Then we calculate the energy-density fluctuation for $\hat{T}_{\text {Can }}^{\mu \nu}$ as

$$
\begin{array}{r}
\sigma_{\text {Can }}^{2}(a, m, T)=2 \int d K d K^{\prime} f\left(\omega_{\boldsymbol{k}}\right)\left(1-f\left(\omega_{\boldsymbol{k}^{\prime}}\right)\right) \times\left[\left(\omega_{\boldsymbol{k}}+\omega_{\boldsymbol{k}^{\prime}}\right)^{2}\left(\omega_{\boldsymbol{k}} \omega_{\boldsymbol{k}^{\prime}}+\boldsymbol{k} \cdot \boldsymbol{k}^{\prime}+m^{2}\right) e^{-\frac{a^{2}}{2}\left(\boldsymbol{k}-\boldsymbol{k}^{\prime}\right)^{2}}\right. \\
\left.-\left(\omega_{\boldsymbol{k}}-\omega_{\boldsymbol{k}^{\prime}}\right)^{2}\left(\omega_{\boldsymbol{k}} \omega_{\boldsymbol{k}^{\prime}}+\boldsymbol{k} \cdot \boldsymbol{k}^{\prime}-m^{2}\right) e^{-\frac{a^{2}}{2}\left(\boldsymbol{k}+\boldsymbol{k}^{\prime}\right)^{2}}\right],
\end{array}
$$

where $d K \equiv d^{3} k /\left((2 \pi)^{3} 2 \omega_{\boldsymbol{k}}\right)$. In Eq. 9, we neglect a temperature-independent term to remove all vacuum divergences [44]. 


\section{2 de Groot-van Leeuwen-van Weert framework}

The de Groot-van Leeuwen-van Weert form of energy-momentum tensor is given as [56]

$$
\hat{T}_{\mathrm{GLW}}^{\mu \nu}=\frac{1}{4 m}\left[-\bar{\psi}\left(\partial^{\mu} \partial^{v} \psi\right)+\left(\partial^{\mu} \bar{\psi}\right)\left(\partial^{v} \psi\right)+\left(\partial^{v} \bar{\psi}\right)\left(\partial^{\mu} \psi\right)-\left(\partial^{\mu} \partial^{v} \bar{\psi}\right) \psi\right] .
$$

In this case we obtain expressions for the thermal average and fluctuation as

$$
\begin{aligned}
\left\langle: \hat{T}_{\mathrm{GLW}, a}^{00}:\right\rangle & =4 \int \frac{d^{3} k}{(2 \pi)^{3}} \omega_{\boldsymbol{k}} f\left(\omega_{\boldsymbol{k}}\right) \equiv \varepsilon_{\mathrm{GLW}}(T) \\
\sigma_{\mathrm{GLW}}^{2}(a, m, T) & =\frac{1}{2 m^{2}} \int d K d K^{\prime} f\left(\omega_{\boldsymbol{k}}\right)\left(1-f\left(\omega_{\boldsymbol{k}^{\prime}}\right)\right)\left[\left(\omega_{\boldsymbol{k}}+\omega_{\boldsymbol{k}^{\prime}}\right)^{4}\left(\omega_{\boldsymbol{k}} \omega_{\boldsymbol{k}^{\prime}}-\boldsymbol{k} \cdot \boldsymbol{k}^{\prime}+m^{2}\right)\right. \\
& \left.\times e^{-\frac{a^{2}}{2}\left(\boldsymbol{k}-\boldsymbol{k}^{\prime}\right)^{2}}-\left(\omega_{\boldsymbol{k}}-\omega_{\boldsymbol{k}^{\prime}}\right)^{4}\left(\omega_{\boldsymbol{k}} \omega_{\boldsymbol{k}^{\prime}}-\boldsymbol{k} \cdot \boldsymbol{k}^{\prime}-m^{2}\right) e^{-\frac{a^{2}}{2}\left(\boldsymbol{k}+\boldsymbol{k}^{\prime}\right)^{2}}\right]
\end{aligned}
$$

respectively. We again discard a divergent term which is temperature-independent. We note that, even though the thermal averages for the canonical and GLW energy-momentum tensors are the same, $\left\langle: \hat{T}_{\mathrm{Can}, a}^{00}:\right\rangle=\left\langle: \hat{T}_{\mathrm{GLW}, a}^{00}:\right\rangle$, their fluctuations are not, $\sigma_{\mathrm{Can}}^{2}(a, m, T) \neq \sigma_{\mathrm{GLW}}^{2}(a, m, T)$.

\subsection{Hilgevoord-Wouthuysen framework}

The Hilgevoord-Wouthuysen form of energy-momentum tensor is defined as below [57, 58]

$$
\hat{T}_{\mathrm{HW}}^{\mu \nu}=\hat{T}_{\mathrm{Can}}^{\mu \nu}+\frac{i}{2 m}\left(\partial^{v} \bar{\psi} \sigma^{\mu \beta} \partial_{\beta} \psi+\partial_{\alpha} \bar{\psi} \sigma^{\alpha \mu} \partial^{v} \psi\right)-\frac{i}{4 m} g^{\mu \nu} \partial_{\lambda}\left(\bar{\psi} \sigma^{\lambda \alpha} \overleftrightarrow{\partial}_{\alpha} \psi\right)
$$

with $\sigma_{\mu \nu} \equiv(i / 2)\left[\gamma_{\mu}, \gamma_{\nu}\right]$. Here the thermal average and fluctuation are calculated respectively as

$$
\begin{aligned}
& \left\langle: \hat{T}_{\mathrm{HW}, a}^{00}:\right\rangle=4 \int \frac{d^{3} k}{(2 \pi)^{3}} \omega_{\boldsymbol{k}} f\left(\omega_{\boldsymbol{k}}\right) \equiv \varepsilon_{\mathrm{HW}}(T) \\
& \sigma_{\mathrm{HW}}^{2}(a, m, T)=\frac{2}{m^{2}} \int d K d K^{\prime} f\left(\omega_{\boldsymbol{k}}\right)\left[\left(\omega_{\boldsymbol{k}} \omega_{\boldsymbol{k}^{\prime}}+\boldsymbol{k} \cdot \boldsymbol{k}^{\prime}+m^{2}\right)^{2}\left(\omega_{\boldsymbol{k}} \omega_{\boldsymbol{k}^{\prime}}-\boldsymbol{k} \cdot \boldsymbol{k}^{\prime}+m^{2}\right)\right. \\
& \left.\times e^{-\frac{a^{2}}{2}\left(\boldsymbol{k}-\boldsymbol{k}^{\prime}\right)^{2}}-\left(\omega_{\boldsymbol{k}} \omega_{\boldsymbol{k}^{\prime}}+\boldsymbol{k} \cdot \boldsymbol{k}^{\prime}-m^{2}\right)^{2}\left(\omega_{\boldsymbol{k}} \omega_{\boldsymbol{k}^{\prime}}-\boldsymbol{k} \cdot \boldsymbol{k}^{\prime}-m^{2}\right) \times e^{-\frac{a^{2}}{2}\left(\boldsymbol{k}+\boldsymbol{k}^{\prime}\right)^{2}}\right]\left(1-f\left(\omega_{\boldsymbol{k}^{\prime}}\right)\right) .
\end{aligned}
$$

It can be seen easily from Eqs. (8), (11), and (14) that $\varepsilon_{\mathrm{Can}}(T)=\varepsilon_{\mathrm{GLW}}(T)=\varepsilon_{\mathrm{HW}}(T)$, while the fluctuations of : $\hat{T}_{a}^{00}$ : are different for different choices of pseudo-gauge. Eqs. (9), (12), and (15) are used to calculate the fluctuations of the energy-density of the subsystem $S_{a}$ of the larger system $S_{V}$. Both the energy density $(\varepsilon)$ and fluctuation $(\sigma)$ can be extended to incorporate other degeneracy factors such as isospin or color charge degrees of freedom.

\section{Summary}

We have calculated the mathematical expressions for quantum energy-density fluctuations for the subsystems of hot relativistic gas of spin- $\frac{1}{2}$ particles. Our results show that even though the energy-density for all choices of pseudo-gauge are the same, still their fluctuations depend on the forms of pseudo-gauge, which means that the quantum fluctuations are pseudo-gauge dependent [8183] and should be kept in mind during the experimental measurements.

I am grateful to A. Das, W. Florkowski, and R. Ryblewski for their intriguing collaboration. This research was supported in part by the Polish National Science Centre Grants No.

2016/23/B/ST2/00717 and No. 2018/30/E/ST2/00432, and IFJ PAN. 


\section{References}

[1] K. Huang, Statistical Mechanics . Wiley New York, 2nd ed., 1987.

[2] M. Smoluchowski, "Beitrag zur Theorie der Opaleszenz von Gasen im kritischen Zustande," Bulletin international de l'Académie des sciences de Cracovie (1911) 493-502.

[3] S. Jeon and V. Koch, "Charged particle ratio fluctuation as a signal for quark-gluon plasma," Phys. Rev. Lett. 85 (Sep, 2000) 2076-2079.

[4] D. J. Gross, R. D. Pisarski, and L. G. Yaffe, "QCD and Instantons at Finite Temperature," Rev. Mod. Phys. 53 (1981) 43.

[5] S. Haussler, M. Bleicher, and H. Stocker, "Susceptibilities and fluctuations in a Quark-Hadron System with Dynamical Recombination," arXiv:0803.2846 [hep-ph].

[6] C. Herzog and K.-W. Huang, "Boundary Fluctuations and A Reduction Entropy," Phys. Rev. D 95 no. 2, (2017) 021901, arXiv: 1610.08970 [hep-th].

[7] V. Vovchenko, D. V. Anchishkin, M. I. Gorenstein, R. V. Poberezhnyuk, and H. Stoecker, "Critical fluctuations in models with van der Waals interactions," Acta Phys. Polon. Supp. 10 (2017) 753, arXiv: 1610.01036 [nucl-th].

[8] J. Steinheimer, V. Vovchenko, J. Aichelin, M. Bleicher, and H. Stöcker, "Conserved charge fluctuations are not conserved during the hadronic phase," Phys. Lett. B 776 (2018) 32-37, arXiv: 1608.03737 [nucl-th].

[9] D. S. Lohr-Robles, E. Lopez-Moreno, and P. O. Hess, "Quantum Phase Transitions within a nuclear cluster model and an effective model of QCD," arXiv:2107.14379 [nucl-th].

[10] Z. Bai, W.-j. Fu, and Y.-x. Liu, "Identifying the QCD Phase Transitions via the Gravitational Wave Frequency from Supernova Explosion," arXiv:2109.12614 [nucl-th].

[11] L. Fortunato, "Quantum phase transitions in algebraic and collective models of nuclear structure," Prog. Part. Nucl. Phys. 121 (2021) 103891.

[12] X. Li, S. Shu, and J.-R. Li, "The quantum fluctuation in an inhomogeneous background and its influence to the phase transition in a finite volume system," arXiv:2108.12325 [hep-ph].

[13] Z.-C. Yang, Y. Li, M. P. A. Fisher, and X. Chen, "Entanglement phase transitions in random stabilizer tensor networks," arXiv:2107.12376 [cond-mat.stat-mech].

[14] M. Sami and R. Gannouji, "Spontaneous symmetry breaking in the late Universe and glimpses of early Universe phase transitions à la baryogenesis," arXiv:2106.00843 $[\mathrm{gr}-\mathrm{qc}]$.

[15] G. P. de Brito, O. Melichev, R. Percacci, and A. D. Pereira, "Can quantum fluctuations differentiate between standard and unimodular gravity?," arXiv:2105.13886 [gr-qc]. 
[16] R. Kubo, “The Fluctuation-Dissipation Theorem,” Rep. Prog. Phys. 29 (1966) 255.

[17] J. Berges and K. Rajagopal, "Color superconductivity and chiral symmetry restoration at nonzero baryon density and temperature," Nucl. Phys. B 538 (1999) 215-232, arXiv: hep-ph/9804233.

[18] A. M. Halasz, A. D. Jackson, R. E. Shrock, M. A. Stephanov, and J. J. M. Verbaarschot, "On the phase diagram of QCD," Phys. Rev. D 58 (1998) 096007, arXiv: hep-ph/9804290.

[19] M. A. Stephanov, K. Rajagopal, and E. V. Shuryak, "Signatures of the tricritical point in QCD,” Phys. Rev. Lett. 81 (1998) 4816-4819, arXiv: hep-ph/9806219.

[20] M. A. Stephanov, K. Rajagopal, and E. V. Shuryak, "Event-by-event fluctuations in heavy ion collisions and the QCD critical point," Phys. Rev. D 60 (1999) 114028, arXiv: hep-ph/9903292.

[21] Y. Hatta and T. Ikeda, "Universality, the QCD critical / tricritical point and the quark number susceptibility," Phys. Rev. D 67 (2003) 014028, arXiv: hep-ph/0210284.

[22] D. T. Son and M. A. Stephanov, "Dynamic universality class of the QCD critical point," Phys. Rev. D 70 (2004) 056001, arXiv: hep-ph/0401052.

[23] M. Stephanov, "Non-Gaussian fluctuations near the QCD critical point," Phys. Rev. Lett. 102 (2009) 032301, arXiv:0809.3450 [hep-ph].

[24] B. Berdnikov and K. Rajagopal, "Slowing out-of-equilibrium near the QCD critical point," Phys. Rev. D 61 (2000) 105017, arXiv: hep-ph/9912274.

[25] S. Caron-Huot, P. M. Chesler, and D. Teaney, "Fluctuation, dissipation, and thermalization in non-equilibrium $\mathrm{AdS}_{5}$ black hole geometries," Phys. Rev. D 84 (2011) 026012 , arXiv: 1102.1073 [hep-th].

[26] M. Kitazawa, M. Asakawa, and H. Ono, "Non-equilibrium time evolution of higher order cumulants of conserved charges and event-by-event analysis," Phys. Lett. B 728 (2014) 386-392, arXiv:1307.2978 [nucl-th].

[27] M. Bluhm et al., "Dynamics of critical fluctuations: Theory - phenomenology - heavy-ion collisions," Nucl. Phys. A 1003 (2020) 122016, arXiv: 2001.08831 [nucl-th].

[28] J. Goswami, F. Karsch, C. Schmidt, S. Mukherjee, and P. Petreczky, "Comparing conserved charge fluctuations from lattice QCD to HRG model calculations," Acta Phys. Polon. Supp. 14 (2021) 251, arXiv:2011.02812 [hep-lat].

[29] W.-j. Fu, X. Luo, J. M. Pawlowski, F. Rennecke, R. Wen, and S. Yin, "High-order baryon number fluctuations within the fRG approach," 9, 2021. arXiv:2110.00163 [hep-ph].

[30] M. Pradeep, K. Rajagopal, M. Stephanov, and Y. Yin, "Freezing out critical fluctuations," in International Conference on Critical Point and Onset of Deconfinement. 9, 2021. arXiv:2109.13188 [hep-ph]. 
[31] J. Goswami, F. Karsch, S. Mukherjee, P. Petreczky, and C. Schmidt, "Conserved charge fluctuations at vanishing net-baryon density from Lattice QCD," in 19th International Conference on Strangeness in Quark Matter. 9, 2021. arXiv:2109.00268 [hep-lat].

[32] D. Bollweg, J. Goswami, O. Kaczmarek, F. Karsch, S. Mukherjee, P. Petreczky, C. Schmidt, and P. Scior, "Second order cumulants of conserved charge fluctuations revisited I. Vanishing chemical potentials," arXiv:2107.10011 [hep-lat].

[33] C. Schmidt, J. Goswami, G. Nicotra, F. Ziesché, P. Dimopoulos, F. Di Renzo, S. Singh, and K. Zambello, "Net-baryon number fluctuations," in Criticality in QCD and the Hadron Resonance Gas. 1, 2021. arXiv:2101.02254 [hep-lat].

[34] X. Guo, K. A. Milton, G. Kennedy, W. P. McNulty, N. Pourtolami, and Y. Li, "The energetics of quantum vacuum friction. I. Field fluctuations,” arXiv:2108.01539 [hep-th].

[35] E. Lifshitz and I. Khalatnikov, "Investigations in relativistic cosmology," Adv. Phys. 12 (1963) 185-249.

[36] A. H. Guth and S.-Y. Pi, "Fluctuations in the new inflationary universe," Phys. Rev. Lett. 49 (Oct, 1982) 1110-1113.

[37] S. Choudhury and A. Mazumdar, "Primordial blackholes and gravitational waves for an inflection-point model of inflation," Phys. Lett. B 733 (2014) 270-275, arXiv: 1307.5119 [astro-ph.CO].

[38] S. Choudhury, S. Panda, and R. Singh, "Bell violation in the Sky," Eur. Phys. J. C 77 no. 2, (2017) 60, arXiv: 1607.00237 [hep-th].

[39] S. Choudhury, S. Panda, and R. Singh, "Bell violation in primordial cosmology," Universe 3 no. 1, (2017) 13, arXiv: 1612.09445 [hep-th].

[40] F. Gelis and B. Schenke, "Initial State Quantum Fluctuations in the Little Bang," Ann. Rev. Nucl. Part. Sci. 66 (2016) 73-94, arXiv: 1604.00335 [hep-ph].

[41] F. Gelis, G. Giacalone, P. Guerrero-Rodríguez, C. Marquet, and J.-Y. Ollitrault, "Primordial fluctuations in heavy-ion collisions," arXiv: 1907.10948 [nucl-th].

[42] S. Choudhury, "The Cosmological OTOC: A New Proposal for Quantifying Auto-correlated Random Non-chaotic Primordial Fluctuations," Symmetry 13 no. 4, (2021) 599, arXiv:2106.01305 [physics.gen-ph].

[43] L. L. Graef, "Constraining the spectrum of cosmological perturbations from statistical thermal fluctuations," Phys. Lett. B 819 (2021) 136418.

[44] A. Das, W. Florkowski, R. Ryblewski, and R. Singh, "Quantum fluctuations of energy in subsystems of a hot relativistic gas," arXiv:2012.05662 [hep-ph]. 
[45] A. Das, W. Florkowski, R. Ryblewski, and R. Singh, "Pseudogauge dependence of quantum fluctuations of the energy in a hot relativistic gas of fermions," Phys. Rev. D 103 no. 9, (2021) L091502, arXiv:2103.01013 [nucl-th].

[46] A. Das, W. Florkowski, R. Ryblewski, and R. Singh, "Quantum baryon number fluctuations in subsystems of a hot and dense relativistic gas of fermions," arXiv:2105.02125 [nucl-th].

[47] R. Singh, "Quantum fluctuations of baryon number density," in Conference on recent developments in high energy physics and cosmology. 9, 2021. arXiv:2109.08201 [nucl-th].

[48] R. Singh, "A compact formula for the quantum fluctuations of energy," in 19th International Conference on Hadron Spectroscopy and Structure. 10, 2021. arXiv: 2110.02727 [nucl-th].

[49] R. Singh, "Mathematical expressions for quantum fluctuations of energy for different energy-momentum tensors," in 9th Large Hadron Collider Physics Conference. 9, 2021. arXiv:2109.11068 [quant-ph].

[50] S. Coleman, Lectures of Sidney Coleman on Quantum Field Theory. WSP, Hackensack, 12, 2018.

[51] F. W. Hehl, "On the energy tensor of spinning massive matter in classical field theory and general relativity," Reports on Mathematical Physics 9 no. 1, (1976) 55-82.

[52] E. Speranza and N. Weickgenannt, "Spin tensor and pseudo-gauges: from nuclear collisions to gravitational physics," arXiv:2007.00138 [nucl-th].

[53] F. Belinfante, “On the spin angular momentum of mesons," Physica 6 no. 7, (1939) 887-898.

[54] F. Belinfante, "On the current and the density of the electric charge, the energy, the linear momentum and the angular momentum of arbitrary fields," Physica 7 no. 5, (1940) 449-474.

[55] L. Rosenfeld Mem. Acad. Roy. Bel. 18 no. 1, (1940).

[56] S. R. De Groot, Relativistic Kinetic Theory. Principles and Applications. 1980.

[57] J. Hilgevoord and S. Wouthuysen, "On the spin angular momentum of the dirac particle," Nuclear Physics 40 (1963) 1-12.

[58] J. Hilgevoord and E. De Kerf, "The covariant definition of spin in relativistic quantum field theory," Physica 31 no. 7, (1965) 1002-1016.

[59] W. Florkowski, A. Kumar, and R. Ryblewski, "Relativistic hydrodynamics for spin-polarized fluids," Prog. Part. Nucl. Phys. 108 (2019) 103709, arXiv: 1811.04409 [nucl-th].

[60] M. Garbiso and M. Kaminski, "Hydrodynamics of simply spinning black holes \& hydrodynamics for spinning quantum fluids," JHEP 12 (2020) 112, arXiv : 2007.04345

[hep-th]. 
[61] R. Singh, "Boost-invariant description of polarization within hydrodynamics with spin," Acta Phys. Polon. Supp. 13 (2020) 931, arXiv: 2001.05592 [hep-ph].

[62] E. Leader and C. Lorcé, "The angular momentum controversy: What's it all about and does it matter?," Phys. Rept. 541 no. 3, (2014) 163-248, arXiv:1309.4235 [hep-ph].

[63] A. D. Gallegos, U. Gürsoy, and A. Yarom, "Hydrodynamics of spin currents," arXiv:2101.04759 [hep-th].

[64] R. Singh, "Conformal transformations of conservation equations in spin hydrodynamics," Acta Phys. Polon. B 52 (2021) 1081, arXiv: 2104.01009 [hep-ph].

[65] K. Dusling, T. Epelbaum, F. Gelis, and R. Venugopalan, "Role of quantum fluctuations in a system with strong fields: Onset of hydrodynamical flow," Nucl. Phys. A 850 (2011) 69-109, arXiv: 1009.4363 [hep-ph].

[66] T. Epelbaum and F. Gelis, "Fluctuations of the initial color fields in high energy heavy ion collisions," Phys. Rev. D 88 (2013) 085015, arXiv: 1307.1765 [hep-ph].

[67] A. Jaiswal and V. Roy, "Relativistic hydrodynamics in heavy-ion collisions: general aspects and recent developments," Adv. High Energy Phys. 2016 (2016) 9623034, arXiv: 1605.08694 [nucl-th].

[68] W. Florkowski, M. P. Heller, and M. Spalinski, "New theories of relativistic hydrodynamics in the LHC era," Rept. Prog. Phys. 81 no. 4, (2018) 046001, arXiv: 1707.02282 [hep-ph].

[69] P. Romatschke and U. Romatschke, Relativistic Fluid Dynamics In and Out of Equilibrium. Cambridge Monographs on Mathematical Physics. Cambridge University Press, 5, 2019. arXiv: 1712.05815 [nucl-th].

[70] G. Giacalone, P. Guerrero-Rodríguez, M. Luzum, C. Marquet, and J.-Y. Ollitrault, "Fluctuations in heavy-ion collisions generated by QCD interactions in the color glass condensate effective theory," Phys. Rev. C 100 no. 2, (2019) 024905, arXiv: 1902.07168 [nucl-th].

[71] R. S. Bhalerao, "Collectivity in large and small systems formed in ultrarelativistic collisions,” Eur. Phys. J. ST 230 no. 3, (2021) 3, arXiv:2009. 09586 [nucl-th].

[72] F. Becattini and M. A. Lisa, "Polarization and Vorticity in the Quark Gluon Plasma," arXiv:2003.03640 [nucl-ex].

[73] S. Bhadury, J. Bhatt, A. Jaiswal, and A. Kumar, "New developments in relativistic fluid dynamics with spin,” Eur. Phys. J. ST 230 no. 3, (2021) 655-672, arXiv: 2101.11964 [hep-ph].

[74] G. Sarwar, M. Hasanujjaman, T. Bhattacharyya, M. Rahaman, A. Bhattacharyya, and J.-e. Alam, "Nonlinear waves in a hot, viscous and nonextensive quark gluon plasma," arXiv:2109.11166 [nucl-th]. 
[75] T. Dore, L. Gavassino, D. Montenegro, M. Shokri, and G. Torrieri, "Fluctuating relativistic dissipative hydrodynamics as a gauge theory," arXiv:2109.06389 [hep-th].

[76] J. M. Karthein, V. Koch, C. Ratti, and V. Vovchenko, "Constraining the hadronic spectrum and repulsive interactions in a hadron resonance gas via fluctuations of conserved charges," arXiv:2107.00588 [nucl-th].

[77] L. Tinti and W. Florkowski, "Particle polarization, spin tensor and the Wigner distribution in relativistic systems," arXiv:2007.04029 [nucl-th].

[78] C. Cohen-Tannoudji, B. Diu, F. Laloë, and S. R. Hemley, Quantum mechanics: Vol. 3: fermions, bosons, photons, correlations and entanglement. A Wiley-Interscience publication. Wiley, New York, 1977.

[79] C. Itzykson and J. Zuber, Quantum Field Theory. International Series In Pure and Applied Physics. McGraw-Hill, New York, 1980.

[80] T. Evans and D. A. Steer, "Wick's theorem at finite temperature," Nucl. Phys. B 474 (1996) 481-496, arXiv: hep-ph/9601268.

[81] S. Mrowczynski, "Density fluctuations in the quark - gluon plasma," Phys. Rev. C 57 (1998) 1518-1521, arXiv:nucl-th/9711051.

[82] F. Becattini and L. Tinti, "Nonequilibrium Thermodynamical Inequivalence of Quantum Stress-energy and Spin Tensors," Phys. Rev. D 87 no. 2, (2013) 025029, arXiv: 1209.6212 [hep-th].

[83] Y. Nakayama, "Intrinsic ambiguity in second order viscosity parameters in relativistic hydrodynamics," Int. J. Mod. Phys. A 27 (2012) 1250125, arXiv: 1206.2421 [hep-th]. 